\title{
Could selected gut microorganisms be diagnostic biomarkers for autism spectrum disorders? Study based on a commercial microbiota test
}

\author{
Magdalena Jendraszak \\ Poznan University of Medical Sciences \\ Mirosława Gałęcka \\ Institute of Microecology \\ Małgorzata Kotwicka \\ Poznan University of Medical Sciences \\ Aleksandra Regdos \\ Institute of Microecology \\ Michalina Pazgrat-Patan \\ Institute of Microecology \\ Mirosław Andrusiewicz ( $\square$ andrus@ump.edu.pl ) \\ Poznan University of Medical Sciences
}

\section{Research Article}

Keywords: gut microbiota, autism spectrum disorders (ASD), gut-brain axis; gastrointestinal problems, dysbiosis

Posted Date: April 9th, 2021

DOI: https://doi.org/10.21203/rs.3.rs-396991/v1

License: (c) (1) This work is licensed under a Creative Commons Attribution 4.0 International License. Read Full License 


\section{Abstract}

The early-life modifications of intestinal microbiota may impact children's subsequent emotional and cognitive development. Stool samples of three groups of children (including probiotics and nonprobiotics) were analyzed. In cases where probiotics were not used, Bifidobacterium spp. levels differed between the ASD and ALG. In healthy non-probiotics use children, strong positive correlations were observed in E. coli and Enterococcus spp. and Bacterioides and Klebsiella spp. In the non-probiotic use ASD group, a strongly negative correlation was observed in Lactobacillus spp., and Faecalibacterium prausnitzii and Akkermansia muciniphila levels. The presented results did not show conclusive evidence that changes in the level of selected intestinal microorganisms are symptomatic for children with ASD.

\section{Introduction}

The human gut microbiota is a complex, non-homogenous ecosystem represented by $10^{13}-10^{14}$ microbes, with over a thousand different species, which possess a hundred fold more genes than found in the human genome. Strict anaerobic bacteria are the primary microcrobes found in the gut, but protozoa, fungi, archaea, and viruses are also detectable. Microbiological colonization of the intestines begins during childbirth. The type of delivery (vaginal or Caesarean section) and gestational age of birth (pre-term or full-term) may play a significant role in post-natal development, as well as in the maturation of endocrine, immune, and nervous systems ${ }^{2-4}$. According to recent studies, early-life modifications of intestinal microbiota may affect subsequent emotional and cognitive development. The diversity of gut microbiota may be crucial for the successful implementation of behavioral skills and proper brain development ${ }^{5-7}$.

In recent years, an increasing number of studies reported that gut microbiota might participate in the process of maintaining human homeostasis through the regulation of mood and well-being, involvement in enteric and central nervous system development, and controlling appetite and metabolism. A bidirectional communication pathway exists between intestinal microorganisms and the brain, known as the gut-brain axis, enabling gut microbes to communicate with the brain, while also acting in an inverse manner. The gut-brain communication mechanisms are complex and have not yet been completely defined. They are assumed to involve many different axes, including immune, neural, endocrine, and metabolic pathways. It is suggested that communication between gut microbiota and the brain occurs through the vagus nerve, the immune system, gut hormone signaling, or microbial metabolites, including short-chain fatty acids (SCFA) and neurotransmitter molecules. It has been demonstrated that gut microbiota might directly affect the brain by production of several bacterial molecules essential for brain functioning e.g.: tryptophan, serotonin, dopamine, kynurenine, and $\mathrm{y}$-aminobutyric acid $^{6-11}$.

However, both clinical and experimental evidence have shown that the homeostatic disorder of gastrointestinal microbiota (dysbiosis) can be related to a wide range of various types of nonneurological diseases e.g.: type 2 diabetes, inflammatory bowel disease, allergies, necrotizing enterocolitis in infants, infections, and obesity ${ }^{12-15}$. On the other hand, dysbiosis can lead to 
neurological changes such as autism, schizophrenia or Parkinson's disease ${ }^{6,16}$ and can impact the severity of psychiatric disorders including depression, stress or anxiety $8,17,18$.

Autism spectrum disorders (ASD), referenced by neurological and developmental dysfunction, are manifested by deficiencies in social communication skills, lack of reciprocal social interactions, and unusual repetitive behaviors. Generally, ASD includes different developmental disorders: the classic form of autistic disorder, Asperger's Syndrome, and Pervasive Developmental Disorder - Not Otherwise Specified ${ }^{19}$. Various studies have shown that genetic implications and environmental factors (chemicals, drugs, diet, prenatal viral infections) can be associated with ASD etiopathogenesis ${ }^{20-22}$. Moreover, according to recent findings, the abundance of some bacterial species, as well as intestinal microbiota composition, may play a crucial role in ASD development and gastrointestinal (GI) problems, which is characteristic for individuals with autism and can be due to gut dysbiosis. A link between alterations in gut microbiota composition and ASD is not well established ${ }^{23-28}$.

Several studies have reported children with ASD present more frequently with gastrointestinal problems such as abdominal pain, constipation or diarrhea, bloating, and/or gastroesophageal reflux than in healthy individuals. In turn, chronic GI disturbances may also aggravate behavioral problems, such as frustration and aggression, and are speculated to correlate with the severity of autism ${ }^{29-33}$. The cause of these intestinal disorders is unknown. However, they appear to be associated with a disarrangement of gut microbiota, particularly, in the excessive growth of pathogenic bacteria (e.g. Clostridium spp.) and the decrease of beneficial microorganisms (such as Lactobacillus and Bifidobacterium) ${ }^{28,34,35}$. Many studies have demonstrated that the fecal microbiota of autistic children differs significantly from the fecal microbiota of neurotypical children. These results are often inconsistent and are not supported by clinical trials based on a large patient group. Moreover, various reports show that the content of the same species of bacteria may be higher or lower in people with ASD. For example, in research carried out by De Angelis et al., the level of Lactobacillus spp., as well as the Bacteroidetes/Firmicutes ratio, in fecal samples of children with autism was lower compared to healthy participants while Clostridium spp. was overrepresented ${ }^{28,34}$. In contrast, Williams et al. noticed an increased Bacteroidetes/Firmicutes ratio in ASD samples ${ }^{36}$. Conversely, Tomova et al. showed a significant decrease in Bacteroidetes/Firmicutes ratio but an increase in Lactobacillus spp. and Clostridium spp. in autistic children ${ }^{30}$. Other microbes observed in large quantities in the feces of ASD compared to neurotypical children include such species as Akkermansia muciniphila and Prevotella ${ }^{34}$ or Desulfovibrio ${ }^{37}$, is contrary to the lower or unchanged level of Akkermansia muciniphila ${ }^{38,39}$, and Prevotella and Desulfovibrio ${ }^{39}$.

The purpose of this study was to examine selected gut microorganisms, both beneficial and pathogenic, in the feces of three groups of children: healthy, with allergies (ALG), and with ASD. Children with allergies were treated as the positive control group. Multiple studies have reported that gut microbiota of allergic patients shows, similar to ASD children, a significant abnormalities in the composition of gut microorganism ${ }^{40-42}$. In our study, we evaluated 19 gut microorganisms by comparing their composition in ASD subjects to neurotypical children. We also considered differences and similarities between groups, 
trying to determine if microbiota imbalances could be the basis for manifestation of, or a marker for, ASD. Our analysis based on the diagnostic intestinal microbiota test enables the detection and identification of foundation and keystone bacterial species in the intestinal ecosystem. Nowadays, diagnostic tests for gut microorganisms are becoming easily available and increasingly used in commercial diagnostics. In this way, we tried to emphasize the practical application of scientific research and link it with the diagnostic process.

\section{Results}

Taking into consideration the Klebsiella spp. level between ASD, ALG, and healthy group participants, we observed significant differences $(p=0.0055)$ regardless of probiotic usage. The Dunn post-hoc test showed a significantly higher median level of Klebsiella spp. in the healthy group compared to ALG children $(p=0.0199)$, with a wider range in children with allergies. No other differences were found regardless of the bacteria species. Taking into consideration probiotics usage, a significant difference in the Klebsiella spp. level $(\mathrm{p}=0.004)$ was observed. The Dunn post-hoc test showed that the levels differed between the healthy and ALG groups $(p=0.028)$. In children who used probiotics, the difference was not significant ( $p=0.593$, Fig. 1$)$. The range was highest in the ALG group, but the median value was slightly higher in the healthy control.

When probiotics were not administered, there was a significant difference in Bifidobacterium spp. $(\mathrm{p}=$ $0.029)$ in the ASD and ALG group $(p=0.036)$ as determined by the Dunn post-hoc test. When probiotics were used, the difference was not significant $(p=0.278$, Fig. 2$)$. The range and median were highest in the healthy controls.

Agglomeration analysis using Euclidian distances (single linkage analysis) Ward's method, was computed for bacteria in the stool. Additionally, the correlation matrix for factor analysis of principal component loadings for multi-presence, multiple-bacteria species with unrotated factor rotation was computed to obtain homogenous subgroups. As presented in Fig. 3, differences in bacteria species presence, distribution, and coexistence in ASD, ALG and healthy group is shown. Although the joining tree shows similar coexistence of different species of bacteria, however, the two way-clustering differs between the health status in some cases. It is especially evident in the variation level of bacteria marked with red boxes and could point dysbiosis. Bifidobacteria and Bacterioides vary the most, but as shown in Fig. 3, in the ASD and the ALG children's stool, the Faecalibacterium prausnitzii level was highly variable. Factor analysis of principal component loadings for multi-presence, multiple-bacteria species highlights the changes in bacterial coexistence. Some species, due to lack of variation in the subgroups, were excluded from the analysis. The adjacent species and the Voronoi tessellation lines show the different presence of the bacteria in the stool and define adjacent polygons, including all peaks in the output plot. As expected, Lactobacillus spp. and $\mathrm{H}_{2} \mathrm{O}_{2}$ Lactobacillus were in a similar distance, always in the same quadrant and near Bacterioides. Surprisingly, E. coli and $C$. albicans were near in the ALG and ASD group but not in healthy children. Akkermansia muciniphila was distant from Bacterioides in all analyzed 
groups. It was not possible to perform the analysis based on probiotic usage due to an insufficient number of cases in those divisions.

Descriptive statistics as a summary of various microorganism species' concentration investigated in the stool of children were shown in Supplementary materials (Table S1., Table S2., and Table S3.). The Supplementary data show values in the whole group, health status divided, and probiotic usage categorized. The Clostridium difficile and molds, due to a lack of conclusive data for all cases, were excluded from statistical analysis.

In the non-probiotic, healthy control group, the Spearman's rank correlation showed a significant, positive, and strong correlation between E. coli and Enterococcus spp. and Bacterioides and Klebsiella spp. (both, $\mathrm{R}=0.79$ ). A negative correlation was observed in Akkermansia muciphila with both Klebsiella spp. and Bacterioides spp. ( $\mathrm{R}=-0.82$ and $\mathrm{R}=-0.80$, respectively). In healthy children that used probiotics, a significant, strong, and positive correlation was shown in the case of the Bacterioides spp. level vs. Bifidobacterium spp. and Akkermansia muciniphila vs. Faecalibacterium prausnitzii (both, $\mathrm{R}=0.68$ ), and a strong negative correlation of Akkermansia muciniphila level vs. C. albicans ( $\mathrm{R}=-0.7)$.

In the ASD group without probiotics, a moderately positive correlation was observed in Lactobacillus spp. level vs. Bifidobacterium spp. $(\mathrm{R}=0.58)$ and $C$. albicans $(\mathrm{R}=0.44)$. Negative correlations were observed in Akkermansia muciniphila vs. both Bifidobacterium spp. and Lactobacillus spp. ( $\mathrm{R}=-0.44$ and $\mathrm{R}=-0.49$, respectively). C. albicans negatively correlated with Faecalibacterium prausnitzii ( $\mathrm{R}=-0.47)$. In the ASD group taking probiotics, a strongly negative correlation was observed in the case of Lactobacillus spp. vs. both Faecalibacterium prausnitzii and Akkermansia muciniphila ( $\mathrm{R}=-0.78$ and $\mathrm{R}=-0.64$, respectively). Additionally, the level Akkermansia muciniphila positively correlated with Faecalibacterium prausnitzii (R $=0.59$ ).

Assessment of the ALG group who did not take probiotics showed a significant, moderate, and positive correlation in the case of Bacterioides spp. and Lactobacillus spp. $(\mathrm{R}=0.48)$ as well as with $C$. albicans vs. both Citrobacterspp. and Clostridium spp. $(\mathrm{R}=0.49$ and $\mathrm{R}=0.63$, respectively). A negative correlation was observed in the case of Faecalibacterium prausnitzii vs. Lactobacillus $(\mathrm{R}=-0.53)$. In the ALG group with probiotics, Enterococcus spp. positively correlated with Lactobacillus spp. $(\mathrm{R}=0.90)$, and a negative correlation was observed in both Akkermansia muciniphila vs. Bifidobacterium spp. and Bacterioides spp. vs. C. albicans ( $\mathrm{R}=-0.81$ and $\mathrm{R}=-0.79$, respectively).

\section{Discussion}

Increasing evidence suggests the balance and diversity within the bacterial population are essential in maintaining proper function of the gastrointestinal tract and immune system as well as human homeostasis. On the other hand, there are a wide range of indicators that propose an imbalance of the gut microbial ecosystem may lead to inflammation and immune activation in several disorders such as 
gastrointestinal diseases, cardiovascular disease, metabolic and psychiatric disorders, allergy, or asthma $6,12,15,16,41$.

The pathogenesis of ASD is complex, and apart from genetic factors, environmental, factors such as the intestinal community, may play a key role in the symptomology of ASD. The composition of gut microorganisms that increase susceptibility to autism development, as well as evidence linking autism symptoms and intestinal dysbiosis, have yet to be fully explained ${ }^{19-21,25,43}$. However, frequent occurrence of GI symptoms in ASD children suggest the involvement of the gut microbiota in gastrointestinal pathophysiology which then constitute potential diagnostic and therapeutic targets. It was suggested that dietary intervention (gluten-, casein-, and soy-free diet), probiotic/prebiotic treatment, microbiota transfer therapy, or targeted antibiotic therapy could be a new strategy for treatment. It could help children with chronic gastrointestinal disorders and may reduce ASD symptoms by improving language, cognitive skills, and behavioral deficits (Doenyas 2018; Fattorusso, Di Genova, Dell'isola, et al. 2019; Kang et al. 2019; $\mathrm{Ng}$ et al. 2019).

Recent studies examining the association of microbiota and children with autism suggest excessive use of antibiotics in ASD individuals may cause an overgrowth of certain Clostridum species such as $C$. tetani, $C$. perfringens, or $C$. difficile. According to the hypothesis linking the occurrence of Clostridium with the etiopathogenesis of autism, an overgrowth of some toxin-producing Clostridum species can expose ASD children to high levels of microbial neurotoxic metabolites. This thereby affects normal nervous system development and leads to the exacerbation of gastrointestinal problems (Argou-Cardozo and Zeidán-Chuliá 2018; Ding et al. 2017; Fattorusso, Di Genova, Dell'Isola, et al. 2019; Finegold et al. 2017; Strati et al. 2017).

Moreover, the anaerobic bacteria Clostridium and Bacteroides are sources of short-chain fatty acids (SCFA), such as propionic, acetic, butyric, and valeric acid, usually produced during fiber fermentation. These metabolites are believed to be involved in gut immune system function, modulation of the nervous system through the gut-brain axis, and host cell gene expression ${ }^{26,34,51}$. SCFAs can induce widespread effects on the human organism, but an imbalance in their levels may change intestinal homeostasis and cause peripheral inflammation. SCFAs reach the brain through blood circulation and affect its development by modulating production of serotonin and dopamine. High concentrations of propionic acid, a significant neurotoxic metabolite, may disrupt brain function, resulting in developmental delay or regression $26,52-54$.

However, the presence of specific Clostridium species, clusters, and their content in the intestinal microbiome of ASD children is still under discussion. Moreover, current results are often inconclusive, and the contribution of selected species in ASD etiology have yet to be fully explained. Our analysis showed no significant differences in the levels of Clostridium spp. within the groups. The results, therefore, are similar to those of Wang and lovene ${ }^{38,55}$ but are not consistent with other reports that found increased Clostridium in the stool of ASD children $34,50,56,57$. 
Bacterioides spp. and Clostridum spp. are defined as bacteria associated with fiber fermentation and SCFA/propionic acid production. It has been suggested that neurodevelopmental disorders in ASD patients correlate with impaired propionic acid metabolism and changes in propionate producing bacteria 54,58 . In our analysis, the level of Bacteroides is unchanged in all analyzed groups. These findings are comparable with those of Parracho et al. and Ma et al. ${ }^{56,59}$ but contrasts other studies where increased Bacteroides in ASD patients has been reported ${ }^{34,51}$. Moreover, in the microbiome of the ASD group, a significant increase of Proteobacteria phylum, particularly species belonging to Enterobacteriaceae, was observed ${ }^{34,51,60}$. However, our analysis showed no significant changes in the abundance of this family in stool samples of any studied groups, except a higher level of Klebsiella species in the healthy group. This result is compatible with Adams' observation ${ }^{29}$.

Additionally, in healthy controls, a significantly positive and strong correlation of Escherichia coli and Enterococcus spp. was noted. It is well-known that certain strains of E. coli and Enterococcus have probiotic properties and can activate the gut mucosal immune system by increasing antibody quantities and cytokine production and also improve the barrier function of the intestinal epithelium ${ }^{61-63}$. Cukrowska et al. has reported the presence of probiotic E. coli Nissle 1917 in infant's intestines may enhance the humoral immune system response, especially the induction of specific IgA and IgM antibodies ${ }^{64}$. Hafez has demonstrated that this beneficial strain may regulate mucin gene expression, thereby altering the intestinal mucus layer and indirectly regulating the gut immune system ${ }^{65}$.

Some studies have also indicated varying levels of probiotic bacteria such as Lactobacillus and Bifidobacterium in the intestines of ASD and neurotypical subjects 27,29,30,34,38,51. Our results showed lower levels of Bifidobacterium in ASD group, which is compatible with several other studies ${ }^{29,34,38,51}$. We speculate this may be due to a derangement of the probiotic bacteria population in the intestines of autistic children. On the other hand, similar numbers of Bifidobacterium in ALG and healthy groups may be a compensatory mechanism. Allergies are a chronic inflammatory diseases, and this group of bacteria shows strong anti-inflammatory properties. Recently published studies have reported that Bifidobacterium strains may inhibit the inflammatory response and exert an immunomodulatory effect by stimulating IL-10 or IL-12 synthesis by dendritic cells ${ }^{66,67}$. Furthermore, the presence of both probiotic bacteria in the intestines contributes to maintenance of the epithelial barrier integrity and protects against an overgrowth of pathogens 55,68 . Additionally, they can impact the metabolism of toxins, drugs, and some dietary compounds as well as gut epithelial cell proliferation ${ }^{68-70}$. Interestingly, both genera may produce $y$-aminobutyric acid (GABA), the primary inhibitory neurotransmitter in the brain ${ }^{26,69}$. According to some studies, lower GABA levels are correlated with anxiety and social disorders in ASD individuals 71,72 .

Moreover, some Lactobacillus and Bifidobacterium strains are the main components of probiotic supplements. Growing clinical evidence suggests the consumption of oral probiotics reduce $\mathrm{GI}$ discomfort, modulate the stress response, and improve mood and anxiety symptoms in patients with ASD 29,30,73,74. However, in our analysis, we observed a strong negative correlation between probiotic 
bacteria and Akkermansia muciniphila and Faecalibacterium prausnitzii levels in ALG and ASD groups using probiotics. We assume this may be due to the dominant role of some probiotic strains or as a result of nutrient competition. Both A. muciniphila and F. prausnitzii are considered biomarkers of healthy intestinal flora and modulators of immune system ${ }^{75,76}$. Additionally, Faecalibacterium may regulate the expression of interferon-gamma (IFNY), which plays an indirect role in neuroplasticity and synapse formation ${ }^{26,77}$. Based on these factors, it can be assumed in children with these associated diseases, selection of appropriate probiotic strains is important, and probiotic therapy should be performed on the basis of previous microbiota analysis.

In our studies, we estimated the content of fecal fungi, especially Candida genus, in ASD children. The healthy gut is colonized by yeast and good bacteria living in balance with one other. Most Candida species are harmless commensals, but when intestinal homeostasis is disturbed, they can cause infections called candidiasis. Yeast infections have been rarely investigated in autistic individuals. Our studies have shown no significant differences between groups. However, some investigators report substantial growth of Candida, particularly Candida albicans, in ASD patients $27,55,78,79$, Contrary to these results, Adams et al. did not confirm these findings ${ }^{29}$. The potential role of the Candida species in ASD etiology is unclear, and further studies are needed. It is believed that an overgrowth of Candida spp. may induce autistic behavior through excessive production of ammonia which then is converted to betaalanine, a non-essential amino acid structurally similar to the inhibitory neurotransmitter GABA ${ }^{79,80}$. Additionally, a high abundance of yeast may impair the absorption of both carbohydrates and mineral elements, as well as affect the release and accumulation of toxins ${ }^{78,80}$. Moreover, enormous growth of Candida in the gut of autistic individuals may aggravate $\mathrm{Gl}$ abnormalities by dysregulation of cytokine release 27 .

\section{Conclusions}

The results of our study do not fully support the hypothesis that the composition of the gastrointestinal microbiota, the presence of certain species, or significantly altered ratios of these microbes change susceptibility to ASD development of children. The formation of intestinal microorganisms is influenced by many factors such as the type of delivery or feeding, child's diet later in life, and even geographical location. Typical research methods are often heterogeneous and do not include this additional information. However, it cannot be excluded that ASD etiopathogenesis is likely multifactorial and involves multiple etiopathogenic mechanisms. Despite the complexity of this issue, it can be assumed that the increased abundance of certain harmful bacterial species, as well as reduction of beneficial ones, in autistic individuals may result in intensified gastrointestinal problems. For these reasons, an analysis of intestinal microbiota along with an exclusion diet enriched with probiotic/prebiotic supplementation could help alleviate GI symptoms and improve the quality of life of ASD children.

\section{Materials And Methods}




\section{Participants}

This study aimed at comparing 89 stool specimens from children who were enrolled to gut microbiota test at the Institute of Microecology (Poznan). The children's parents completed a self-reporting questionnaire consisting of a set of questions regarding their child's: age, sex, body mass, height, health status, probiotic or/and antibiotic supplementation, and radiotherapy/chemotherapy treatment. Based on this information, three children's groups were distinguished: without existing illnesses, with allergies, and with ASD. The individuals with autoimmune diseases, Lyme disease, diabetes, cancer, and children who had undergone antibiotic therapy within three months, radiotherapy, and chemotherapy were excluded from further analysis. Neurotypical children and allergy sufferers were not related to any degree with ASD children. The children were enrolled regardless of any gastrointestinal disturbances. According to the exclusion criteria, 73 children were included in further statistical analysis: 16 healthy children, 24 with allergies, and 33 with ASD. In the ASD group, 9 out of 33 children (27\%) and 7 out of 27 (29\%) of ALG group reported gastrointestinal symptoms, while none of the children in the healthy group. The descriptive statistics for the study group was shown in Table 1. The healthy children and those with allergic diseases were treated as negative and positive controls, respectively. The data were analyzed between the illness status groups as well as probiotic usage. Informed consent was obtained from all parents or legally authorized representatives, and identifying information was removed from each sample. The study protocol was in accordance with the Declaration of Helsinki and was approved by the Ethical Committee of Poznan University of Medical Sciences.

Table 1

Summary of subject characteristics

\begin{tabular}{|llll|}
\hline & Healthy & ASD & ALG \\
\hline Total participants $^{\mathrm{a}}$ & $16(22 \%)$ & $33(45 \%)$ & $24(33 \%)$ \\
\hline Female/Male $^{\mathrm{a}}$ & $6(38 \%) / 10(63 \%)$ & $4(12 \%) / 29(88 \%)$ & $9(38 \%) / 15(62 \%)$ \\
\hline Age (year) $^{\mathrm{b}}$ & $5.5[3-9]$ & $5[4-6]$ & $7[4.5-9.5]$ \\
\hline BMI $^{\mathrm{b}}$ & $14.88[14.20-16.97]$ & $14.86[14.06-16.02]$ & $15.16[14.18-18.07]$ \\
\hline Probiotic usage [yes/no] $^{\mathrm{a}}$ & $9(56 \%) / 7(44 \%)$ & $12(36 \%) / 21(64 \%)$ & $7(29 \%) / 17(71 \%)$ \\
\hline Gastrointestinal disorders $^{\mathrm{a}}$ & 0 & $9(27 \%)$ & $7(29 \%)$ \\
\hline $\begin{array}{l}\text { Legend: ALG - Allergies; ASD } \\
\text { cases (percentage); }{ }^{\mathrm{b}}-\text { Median }^{\text {[interquartile range] }}\end{array}$ & & \\
\hline
\end{tabular}

\section{Materials and Procedures}


Stool samples were collected in sterile stool tubes at the participants' homes and delivered to the Institute of Microecology (Poznan), where they were analyzed for selected intestinal microorganisms. The analysis was carried out following the KyberKompakt Pro protocol and included both microbiological cultures and quantitative polymerase chain reactions (qPCR). All counts were expressed as the numbers of $\log _{10} \mathrm{CFU}$ (colony-forming unit) per $1 \mathrm{~g}$ of sample.

\section{Microbiological identification of selected microorganisms}

Before microbial culture, $0.25 \mathrm{~g}$ of each sample was diluted ten times in $0.85 \%$ sterile $\mathrm{NaCl}$ solution, suspended by vortexing, and subsequently plated on selective and differential agar medium plates.

The viable bacterial cells in feces were inoculated on the following selective media: Columbia blood agar (total bacteria count; Becton Dickinson, Heidelberg, Germany), Chromid CPS agar (Escherichia coli, Proteus, Enterococcus and Klebsiella, Enterobacter, Serratia and Citrobacter; BioMerieux, Durham, USA), Rogosa TMB HPR agar (Lactobacillus; Heipha, Eppelheim, Germany), Bifidobacterium agar (Bifidobacteria; Becton Dickinson, Germany), Schaedler agar (Bacteroides; Heipha, Eppelheim, Germany), and SPM agar (Clostridium spp.; Heipha, Eppelheim, Germany). The plates were incubated under either aerobic or anaerobic conditions at $37 \mathrm{C}^{\circ}$ for 24 and $48 \mathrm{~h}$.

Both cultures and microscopic observations determined the presence of fecal fungi. Samples were suspended in $0.85 \%$ sterile $\mathrm{NaCl}$ solution containing trypsin and antibiotics (penicillin-streptomycin). Afterward, samples were inoculated into two Sabouraud agar plates with antibiotics (gentamicin and chloramphenicol). After 2-5 days, yeast colonies were transferred to differential plates were assigned to the taxonomy species group. Molds were examined by morphological observation after 5-7 days of incubation.

To analyze anaerobic, unculturable bacteria such as Akkermansia muciniphila and Faecalibacterium prausnitzii and determine Clostridium difficile numbers, quantitative polymerase chain reaction was used.

\section{DNA isolation and quantitative PCR analysis}

Bacterial DNA from stool samples was extracted using RIDA® Xtract kit in accordance with the manufacturer's instructions. To estimate bacterial quantity, qPCR was performed with the use of RIDA®GENE Akkermansia muciniphila, RIDA®GENE Faecalibacterium prausnitzii, and RIDA®GENE Clostridium difficile kits in accordance with the manufacturer's instructions (R-Biopharm AG, Darmstadt, Germany). The thermal profile was as follows: initial denaturation $\left(1 \mathrm{~min}, 95^{\circ} \mathrm{C}\right)$, then 45 cycles of denaturation $\left(15 \mathrm{sec}, 95^{\circ} \mathrm{C}\right)$ and annealing/extension $\left(30 \mathrm{sec}, 60^{\circ} \mathrm{C}\right)$. The total reaction mixture volume was $25 \mu \mathrm{L}$ containing $19.9 \mu \mathrm{L}$ reaction mix, $0.1 \mu \mathrm{L}$ Taq Polymerase, and $5 \mu \mathrm{L}$ DNA-extract. The standard curve was generated with standard DNA A: $5 \times 10^{2}$ copies/reaction, standard DNA B: $5 \times 10^{4}$ copies/reaction, and standard DNA C: $5 \times 10^{6}$ copies/reaction. The reaction was performed in the RotorGene thermal cycler (QIAGEN, Manheim, Germany). The final number of bacteria/gram of stool was obtained by multiplying by 200 due to the dilution factor of the stool sample during extraction.

\section{Statistical analyses}

Page $10 / 19$ 
Several statistical analyses were performed using Statistica ver. 13 software (TIBCO Software, Tulsa, USA). The distributions of the continuous variables were assessed with the Shapiro-Wilk test. As the data was not normally distributed, a nonparametric, 2-sided Kruskal-Wallis test with Dunn's post-hoc test for multiple comparisons was used. Nonparametric Spearman's rank correlation test was applied to determine the strength of a link between microbe species. For individual comparisons, a p-value of $<0.05$ was considered significant. The results were described using nonparametric descriptive statistics. The correlation matrix for factor analysis of principal component loadings for multi-presence, multiplebacteria strains was computed to determine homogenous subgroups. It was calculated for 12 strains with unrotated factor rotation. Missing data were case wise deleted, 80 cases were processed, and 73 valid cases were accepted. Based on the multi-presence of microorganisms, using Ward's method cluster joining and Euclidean distances, the bacteria genera were clustered.

\section{Declarations:}

\section{Author contributions}

Conceptualization, M.J.; methodology, M.A., MP-P., A.R.; validation, M.J., M.K., M.A., A.R., and M.G.; formal analysis, M.J., M.A., A.R. and M.P-P.; investigation, M.J., M.A., A.R., and M.P-P.; resources, M.A., M.J., M.G., and M.K.; data curation, M.P-P., M.G., M.A., M.P-P. and M.A.; writing-original draft preparation, M.J., M.G., A.R., M.P-P., and M.A.; writing-review and editing, M.J., M.G., A.R., M.P-P., and M.A.; visualization, M.A., and M.J.; supervision, M.J., M.G., and M.K.

\section{Institutional Review Board Statement}

The study was conducted according to the guidelines of the Declaration of Helsinki, and approved by the Institutional Review Board of Poznan University of Medical Sciences.

\section{Informed Consent Statement}

Informed consent was obtained from all parents or legally authorized representatives, and identifying information was removed from each sample.

\section{Acknowledgments}

Not applicable

\section{Conflicts of Interest}

The authors declare no conflict of interest.

\section{References}


1. Sharma, L. K. et al. Mitochondrial respiratory complex I dysfunction promotes tumorigenesis through ROS alteration and AKT activation. Hum. Mol. Genet. 20, 4605-16 (2011).

2. Sekirov, I., Russell, S. L., Caetano M Antunes, L. \& Finlay, B. B. Gut microbiota in health and disease. Physiol. Rev. 90, 859-904 (2010).

3. Christian Milani, Sabrina Duranti, Francesca Bottacini, B. et al. The First Microbial Colonizers of the Human Gut: Composition, Activities, and Health Implications of the Infant Gut Microbiota. Microbiol. Mol. Biol. Rev. 81, 1-67 (2017).

4. Hillman, E. T., Lu, H., Yao, T. \& Nakatsu, C. H. Microbial ecology along the gastrointestinal tract. Microbes Environ. 32, 300-313 (2017).

5. Rogers, G. B. et al. From gut dysbiosis to altered brain function and mental illness: Mechanisms and pathways. Mol. Psychiatry 21, 738-748 (2016).

6. Dinan, T. G. \& Cryan, J. F. Gut instincts: microbiota as a key regulator of brain development, ageing and neurodegeneration. J. Physiol. 595, 489-503 (2017).

7. Mayer, E. a et al. Gut/brain axis and the microbiota Emeran. Nutr. Cancer 125, 463-479 (2015).

8. Foster, J. A. \& McVey Neufeld, K. A. Gut-brain axis: How the microbiome influences anxiety and depression. Trends Neurosci. 36, 305-312 (2013).

9. Carabotti, M., Scirocco, A., Maselli, M. A. \& Severi, C. The gut-brain axis: Interactions between enteric microbiota, central and enteric nervous systems. Ann. Gastroenterol. 28, 203-209 (2015).

10. van de Wouw, M., Schellekens, H., Dinan, T. G. \& Cryan, J. F. Microbiota-Gut-Brain Axis: Modulator of Host Metabolism and Appetite. J. Nutr. 147, 727-745 (2017).

11. Microbial Gut-Brain Axis in The Microbiota-Endocrinology: Health and Disease. Advances in Experimental Medicine and Biology (Springer, 2014). doi:10.1007/978-1-4939-0987-4

12. Carding, S., Verbeke, K., Vipond, D. T., Corfe, B. M. \& Owen, L. J. Dysbiosis of the gut microbiota in disease. Microb. Ecol. Heal. Dis. 26, (2015).

13. Scorletti, E. \& Byrne, C. D. Extrahepatic Diseases and NAFLD: The Triangular Relationship between NAFLD, Type 2-Diabetes and Dysbiosis. Dig. Dis. 34, 11-18 (2016).

14. Pammi, M. et al. Intestinal dysbiosis in preterm infants preceding necrotizing enterocolitis: A systematic review and meta-analysis. Microbiome 5, 1-15 (2017).

15. Icaza-Chávez, M. E. Gut microbiota in health and disease. Rev. Gastroenterol. México (English Ed. 78, 240-248 (2013).

16. Dinan, T. G. \& Cryan, J. F. The impact of gut microbiota on brain and behaviour: Implications for psychiatry. Curr. Opin. Clin. Nutr. Metab. Care 18, 552-558 (2015).

17. Luna, R. A. \& Foster, J. A. Gut brain axis: Diet microbiota interactions and implications for modulation of anxiety and depression. Curr. Opin. Biotechnol. 32, 35-41 (2015).

18. Vuong, H. E., Yano, J. M., Fung, T. C. \& Hsiao, E. Y. The Microbiome and Host Behavior. Annu. Rev. Neurosci. 40, 21-49 (2017). 
19. Faras, H., Al Ateeqi, N. \& Tidmarsh, L. Autism spectrum disorders. Ann. Saudi Med. 30, 295-300 (2010).

20. Grabrucker, A. M. Environmental factors in autism. Front. Psychiatry 3, 1-13 (2013).

21. Dietert, R. R., Dietert, J. M. \& DeWitt, J. C. Environmental risk factors for autism. Emerg. Health Threats J. 4, 1-11 (2011).

22. Modabbernia, A., Velthorst, E. \& Reichenberg, A. Environmental risk factors for autism: an evidencebased review of systematic reviews and meta-analyses. Mol. Autism 8, 1-16 (2017).

23. Hughes, H. K., Rose, D. \& Ashwood, P. The Gut Microbiota and Dysbiosis in Autism Spectrum Disorders. Curr. Neurol. Neurosci. Rep. 18, (2018).

24. Heberling, C. A., Dhurjati, P. S. \& Sasser, M. Hypothesis for a systems connectivity model of autism spectrum disorder pathogenesis: Links to gut bacteria, oxidative stress, and intestinal permeability. Med. Hypotheses 80, 264-270 (2013).

25. Finegold, S. M. State of the art; microbiology in health and disease. Intestinal bacterial flora in autism. Anaerobe 17, 367-368 (2011).

26. Srikantha, P. \& Hasan Mohajeri, M. The possible role of the microbiota-gut-brain-axis in autism spectrum disorder. Int. J. Mol. Sci. 20, 14-19 (2019).

27. Strati, F. et al. New evidences on the altered gut microbiota in autism spectrum disorders. Microbiome 5, 1-11 (2017).

28. de Angelis, M., Francavilla, R., Piccolo, M., De Giacomo, A. \& Gobbetti, M. Autism spectrum disorders and intestinal microbiota. Gut Microbes 6, 207-213 (2015).

29. Adams, J. B., Johansen, L. J., Powell, L. D., Quig, D. \& Rubin, R. A. Gastrointestinal flora and gastrointestinal status in children with autism - comparisons to typical children and correlation with autism severity. BMC Gastroenterol. 11, 22 (2011).

30. Tomova, A. et al. Gastrointestinal microbiota in children with autism in Slovakia. Physiol. Behav. 138, 179-187 (2015).

31. Nikolov, R. N. et al. Gastrointestinal symptoms in a sample of children with pervasive developmental disorders. J. Autism Dev. Disord. 39, 405-413 (2009).

32. Kevin Range, and D. M. Y. A. M. Autism Spectrum Disorder: Defining Dimensions and Subgroups. Bone 23, 1-7 (2012).

33. Fulceri, F. et al. Gastrointestinal symptoms and behavioral problems in preschoolers with Autism Spectrum Disorder. Dig. Liver Dis. 48, 248-254 (2016).

34. De Angelis, M. et al. Fecal Microbiota and Metabolome of Children with Autism and Pervasive Developmental Disorder Not Otherwise Specified. PLoS One 8, 1-18 (2013).

35. Ding, H. T., Taur, Y. \& Walkup, J. T. Gut Microbiota and Autism: Key Concepts and Findings. J. Autism Dev. Disord. 47, 480-489 (2017).

36. Williams, B. L. et al. Impaired carbohydrate digestion and transport and mucosal dysbiosis in the intestines of children with autism and gastrointestinal disturbances. PLoS One 6, (2011). 
37. Finegold, S. M. et al. Pyrosequencing study of fecal microflora of autistic and control children. Anaerobe 16, 444-453 (2010).

38. Wang, L. et al. Low relative abundances of the mucolytic bacterium Akkermansia muciniphila and Bifidobacterium spp. in feces of children with autism. Appl. Environ. Microbiol. 77, 6718-6721 (2011).

39. Kang, D. W. et al. Reduced Incidence of Prevotella and Other Fermenters in Intestinal Microflora of Autistic Children. PLoS One 8, (2013).

40. Bisgaard, H. et al. Reduced diversity of the intestinal microbiota during infancy is associated with increased risk of allergic disease at school age. J. Allergy Clin. Immunol. 128, (2011).

41. Bridgman, S. L., Kozyrskyj, A. L., Scott, J. A., Becker, A. B. \& Azad, M. B. Gut microbiota and allergic disease in children. Ann. Allergy, Asthma Immunol. 116, 99-105 (2016).

42. McCoy, K. D. \& Köller, Y. New developments providing mechanistic insight into the impact of the microbiota on allergic disease. Clin. Immunol. 159, 170-176 (2014).

43. Finegold, S. M., Downes, J. \& Summanen, P. H. Microbiology of regressive autism. Anaerobe 18, 260262 (2012).

44. Doenyas, C. Dietary interventions for autism spectrum disorder: New perspectives from the gut-brain axis. Physiol. Behav. 194, 577-582 (2018).

45. Fattorusso, A., Di Genova, L., Dell'isola, G. B., Mencaroni, E. \& Esposito, S. Autism spectrum disorders and the gut microbiota. Nutrients 11, (2019).

46. Ng, Q. X. et al. A systematic review of the role of prebiotics and probiotics in autism spectrum disorders. Med. 55, 1-10 (2019).

47. Kang, D. W. et al. Long-term benefit of Microbiota Transfer Therapy on autism symptoms and gut microbiota. Sci. Rep. 9, 1-9 (2019).

48. Argou-Cardozo, I. \& Zeidán-Chuliá, F. Clostridium Bacteria and Autism Spectrum Conditions: A Systematic Review and Hypothetical Contribution of Environmental Glyphosate Levels. Med. Sci. 6, 29 (2018).

49. Fattorusso, A. et al. crossm Alterations in Gut Glutamate Metabolism Associated with Changes in Gut Microbiota Composition in Children with. Nutrients 14, 61 (2019).

50. Finegold, S. M., Summanen, P. H., Downes, J., Corbett, K. \& Komoriya, T. Detection of Clostridium perfringens toxin genes in the gut microbiota of autistic children. Anaerobe 45, 133-137 (2017).

51. Coretti, L. et al. Gut microbiota features in young children with autism spectrum disorders. Front. Microbiol. 9, 1-12 (2018).

52. Abdelli, L. S., Samsam, A. \& Naser, S. A. Propionic Acid Induces Gliosis and Neuro-inflammation through Modulation of PTEN/AKT Pathway in Autism Spectrum Disorder. Sci. Rep. 9, 1-12 (2019).

53. Frye, R. E. et al. Modulation of immunological pathways in autistic and neurotypical lymphoblastoid cell lines by the enteric microbiome metabolite propionic acid. Front. Immunol. 8, 1-7 (2017). 
54. MacFabe, D. F. et al. Neurobiological effects of intraventricular propionic acid in rats: Possible role of short chain fatty acids on the pathogenesis and characteristics of autism spectrum disorders. Behav. Brain Res. 176, 149-169 (2007).

55. Iovene, M. R. et al. Intestinal Dysbiosis and Yeast Isolation in Stool of Subjects with Autism Spectrum Disorders. Mycopathologia 182, 349-363 (2017).

56. Ma, B. et al. Altered gut microbiota in Chinese children with autism spectrum disorders. Front. Cell. Infect. Microbiol. 9, 1-10 (2019).

57. Kandeel, W. A. et al. Impact of Clostridium Bacteria in Children with Autism Spectrum Disorder and Their Anthropometric Measurements. J. Mol. Neurosci. 70, 897-907 (2020).

58. Wang, L. et al. Elevated fecal short chain fatty acid and ammonia concentrations in children with autism spectrum disorder. Dig. Dis. Sci. 57, 2096-2102 (2012).

59. Parracho, H. M. R. T., Bingham, M. O., Gibson, G. R. \& McCartney, A. L. Differences between the gut microflora of children with autistic spectrum disorders and that of healthy children. J. Med. Microbiol. 54, 987-991 (2005).

60. Li, N. et al. Correlation of Gut Microbiome Between ASD Children and Mothers and Potential Biomarkers for Risk Assessment. Genomics, Proteomics Bioinforma. 17, 26-38 (2019).

61. La Fata, G., Weber, P. \& Mohajeri, M. H. Probiotics and the Gut Immune System: Indirect Regulation. Probiotics Antimicrob. Proteins 10, 11-21 (2018).

62. Ashraf, R. \& Shah, N. P. Immune System Stimulation by Probiotic Microorganisms. Crit. Rev. Food Sci. Nutr. 54, 938-956 (2014).

63. Molina, M. A. et al. Immunostimulatory effects triggered by Enterococcus faecalis CECT7121 probiotic strain involve activation of dendritic cells and interferon-gamma production. PLoS One 10, 1-20 (2015).

64. Cukrowska, B. et al. Specific proliferative and antibody responses of premature infants to intestinal colonization with nonpathogenic probiotic E. coli strain Nissle 1917. Scand. J. Immunol. 55, 204209 (2002).

65. Hafez, M. M. Upregulation of Intestinal Mucin Expression by the Probiotic Bacterium E. coli Nissle 1917. Probiotics Antimicrob. Proteins 4, 67-77 (2012).

66. Akay, H. K. et al. The relationship between bifidobacteria and allergic asthma and/or allergic dermatitis: A prospective study of 0-3 years-old children in Turkey. Anaerobe 28, 98-103 (2014).

67. Khokhlova, E. V. et al. Anti-inflammatory properties of intestinal Bifidobacterium strains isolated from healthy infants. Microbiol. Immunol. 56, 27-39 (2012).

68. Martín, R. et al. Role of commensal and probiotic bacteria in human health: A focus on inflammatory bowel disease. Microb. Cell Fact. 12, 1-11 (2013).

69. Hemarajata, P. \& Versalovic, J. Effects of probiotics on gut microbiota: Mechanisms of intestinal immunomodulation and neuromodulation. Therap. Adv. Gastroenterol. 6, 39-51 (2013).

70. Wieërs, G. et al. How Probiotics Affect the Microbiota. Front. Cell. Infect. Microbiol. 9, (2020). 
71. Puts, N. A. J. et al. Reduced GABA and altered somatosensory function in children with autism spectrum disorder. Autism Res. 10, 608-619 (2017).

72. Coghlan, S. et al. GABA system dysfunction in autism and related disorders: From synapse to symptoms. Neuroscience and Biobehavioral Reviews 36, 2044-2055 (2012).

73. Navarro, F., Liu, Y. \& Rhoads, J. M. Can probiotics benefit children with autism spectrum disorders? World J. Gastroenterol. 22, 10093-10102 (2016).

74. Santocchi, E. et al. Gut to brain interaction in Autism Spectrum Disorders: A randomized controlled trial on the role of probiotics on clinical, biochemical and neurophysiological parameters. $B M C$ Psychiatry 16, 1-16 (2016).

75. Geerlings, S., Kostopoulos, I., de Vos, W. \& Belzer, C. Akkermansia muciniphila in the Human Gastrointestinal Tract: When, Where, and How? Microorganisms 6, 75 (2018).

76. Miquel, S. et al. Faecalibacterium prausnitzii and human intestinal health. Curr. Opin. Microbiol. 16, 255-261 (2013).

77. Iglesias-vázquez, L., Riba, G. V. G., Arija, V. \& Canals, J. Composition of gut microbiota in children with autism spectrum disorder: A systematic review and meta-analysis. Nutrients 12, 1-21 (2020).

78. Kantarcioglu, A. S., Kiraz, N. \& Aydin, A. Microbiota-Gut-Brain Axis: Yeast Species Isolated from Stool Samples of Children with Suspected or Diagnosed Autism Spectrum Disorders and In Vitro Susceptibility Against Nystatin and Fluconazole. Mycopathologia 181, 1-7 (2016).

79. Emam, Esmat \& Sadek. Candida Albicans Infection in Autism. J. Am. Sci. 8, 739-744 (2012).

80. Li, Q., Han, Y., Dy, A. B. C. \& Hagerman, R. J. The gut microbiota and autism spectrum disorders. Front. Cell. Neurosci. 11, (2017).

\section{Figures}



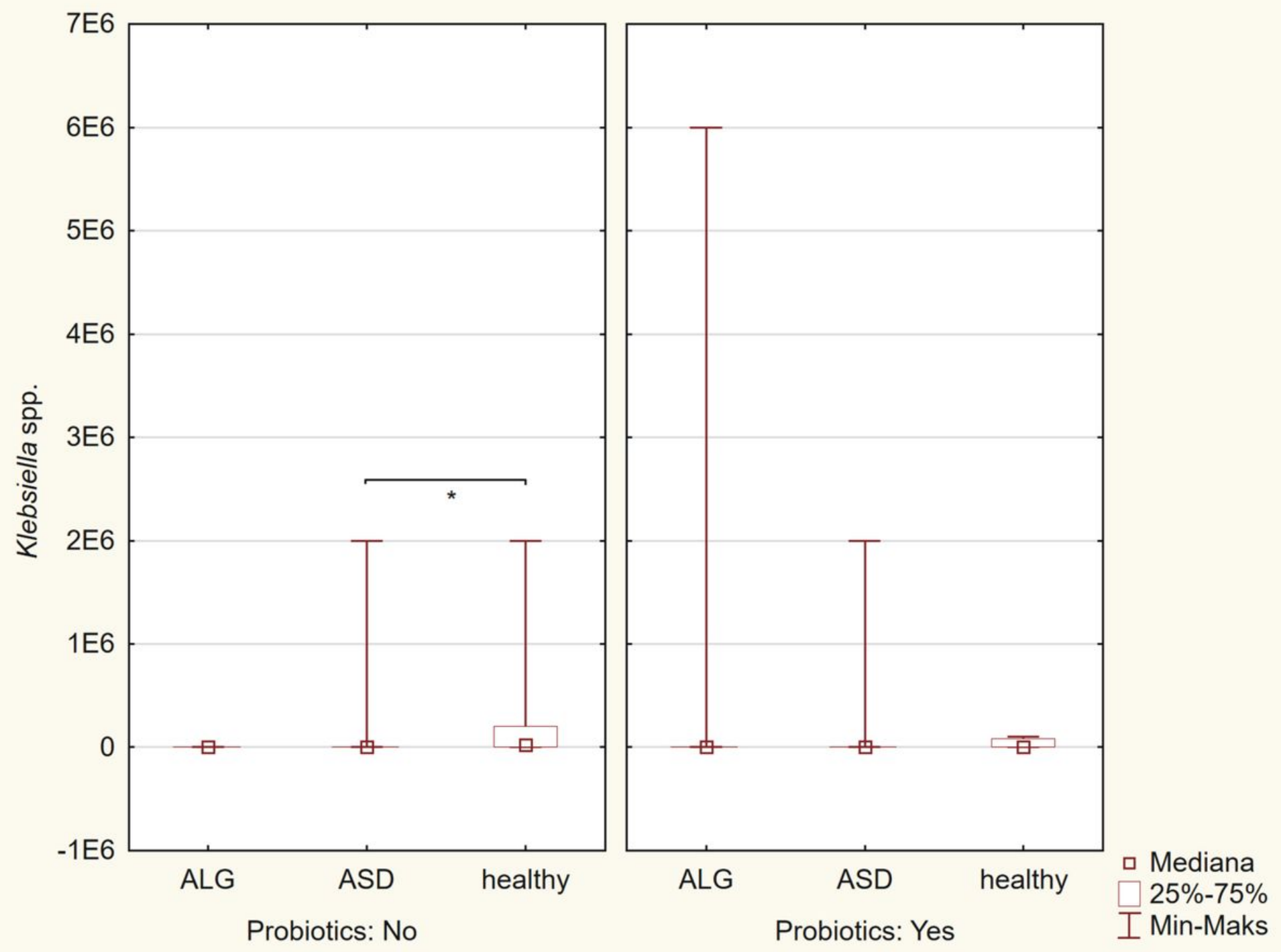

Figure 1

Klebsiella spp. level in the stool of children with ASD, allergies, and in the healthy group divided by probiotic usage 


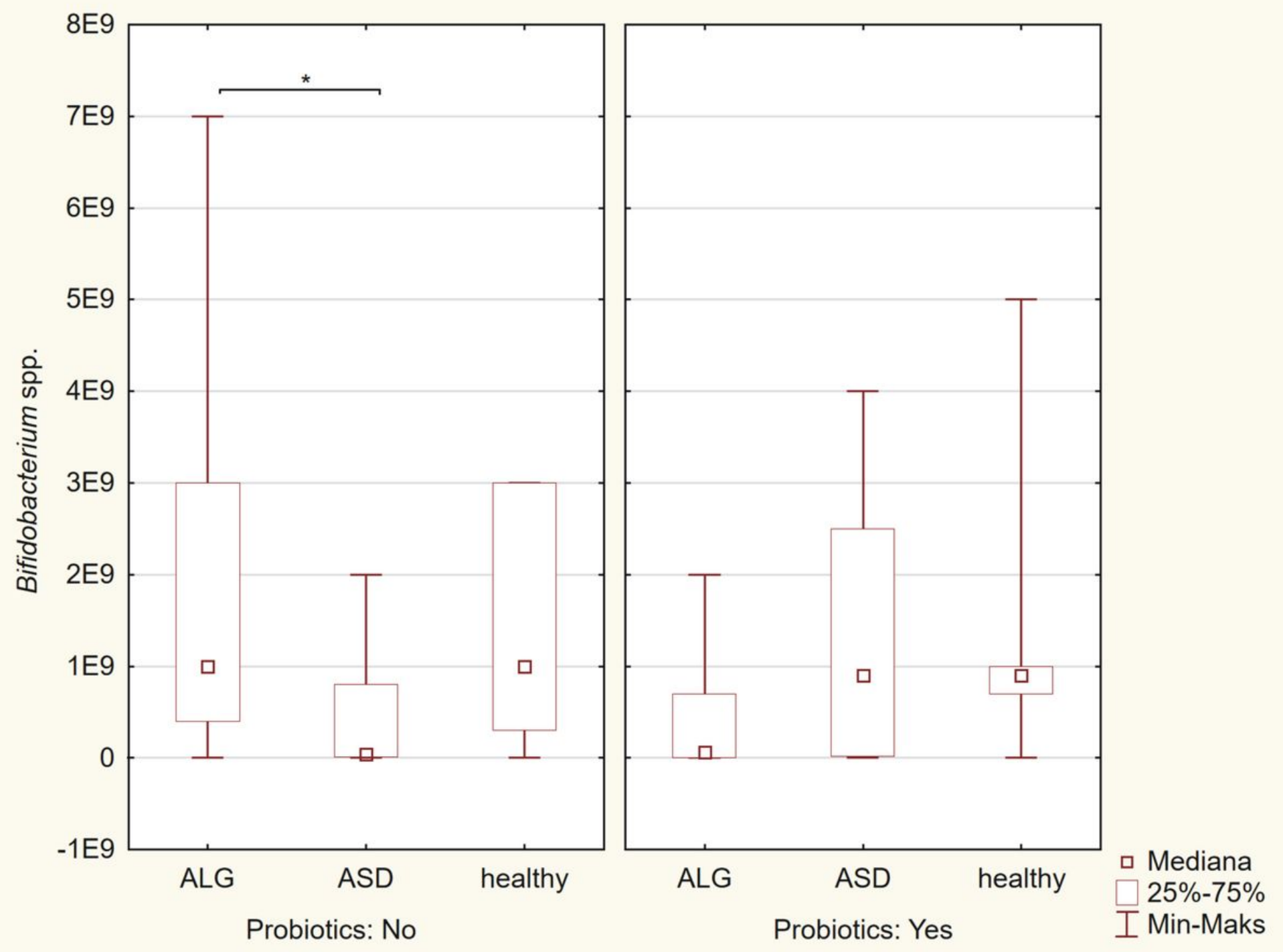

Figure 2

Bifidobacterium spp. level in the stool of children with ASD, allergies, and in the healthy group divided by probiotic usage 

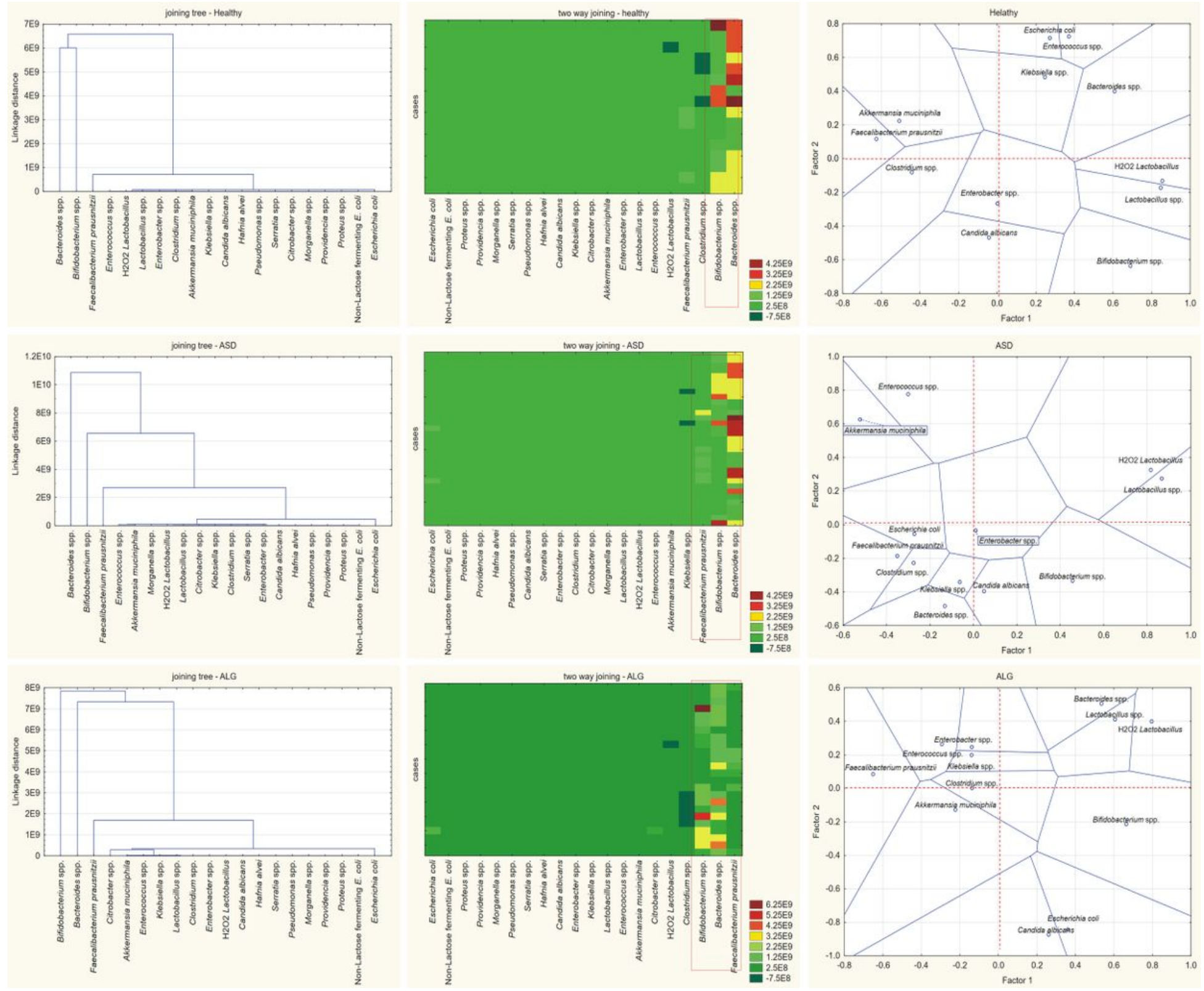

Figure 3

Agglomeration analysis using Euclidian distances, to way joining and correlation matrix for factor analysis of principal components loadings for multi-presence multiple-bacteria species

\section{Supplementary Files}

This is a list of supplementary files associated with this preprint. Click to download.

- 2021.01.08SupplementaryTables.docx 